\title{
COMMENTARY
}

\section{Recognition of hypovolemic shock: using base deficit to think outside of the ATLS box}

\author{
Alicia R Privette* and Rochelle A Dicker \\ See related research by Mutschler et al., http://ccforum.com/content/17/2/R42
}

\begin{abstract}
Base deficit has frequently been utilized as an informal adjunct in the initial evaluation of trauma patients to assess the extent of their physiologic derangements. However, the current Advanced Trauma Life Support (ATLS) classification system for hypovolemic shock does not include base-deficit measurements and relies primarily on alterations in vital signs (heart rate, systolic blood pressure) and mental status (Glasgow Coma Scale) to estimate blood loss. The authors of this paper propose that the current ATLS system may not accurately reflect the degree of hypovolemic shock in many patients and that base-deficit measurements should be used in its place. The proposed system showed a greater correlation with transfusion requirements, need for massive transfusion, and mortality when compared with the ATLS classification system. Based on these findings, base-deficit measurement should be strongly considered during the initial trauma evaluation to identify the presence of hypovolemic shock and to guide blood product administration.
\end{abstract}

The study by Mutschler and colleagues compares the accuracy of the current Advanced Trauma Life Support (ATLS) classification system for hypovolemic shock with a system based on alterations in base deficit [1]. The impetus for this study is recent data questioning the clinical accuracy of the ATLS classification system. The ATLS system currently relies on alterations in vital signs (systolic blood pressure, heart rate) and mental status to estimate the patient's degree of blood loss (class I to IV), and to guide fluid administration [2]. The concern raised by these studies is that the current system's reliance on

*Correspondence: arprivette@gmail.com

San Francisco General Hospital/Univsersity of California San Francisco, 1001 Potrero Avenue, Ward 3A, San Francisco, CA 94110, USA vital signs and mental status may lead to inaccurate assessment and treatment of patients with shock [3,4]. In response to these concerns, the authors have proposed an easily quantifiable measurement of hypovolemic shock based solely on initial base deficit.

The current ATLS system was designed to standardize the initial management of severely injured trauma patients and has become the unofficial gold standard worldwide. A key focus of initial management is the early recognition and treatment of hypovolemic shock. Delayed treatment of hypovolemic shock has been linked to adverse outcomes, including increased organ dysfunction and mortality [5]. Thus, for each class of shock (class I to IV), ATLS recommends a specific intervention (crystalloid \pm blood) to mitigate the presumed level of hemorrhage. However, multiple studies have shown that the ATLS classification system may not accurately reflect the degree of hemorrhage. Two studies by Guly and colleagues demonstrated that inter-relationships existed between components (heart rate, systolic blood pressure, respiratory rate, Glasgow Coma Scale) of the ATLS system, but these associations did not correlate to the levels used to define the ATLS classes of shock [3,4]. These findings, in combination with an earlier report by Mutschler and colleagues [6], suggest that the current system may overestimate the degree of tachycardia in relation to hypotension and underestimate mental status changes. In addition, individual components of the system appear to be poor predictors of hemorrhage. For example, tachycardia (one of the earliest signs of shock in the ATLS system) has been shown to be an inaccurate predictor of hypovolemic shock in several studies $[7,8]$. The ATLS classification system, either in part or as a whole, may not accurately reflect a patient's degree of hypovolemic shock. This discrepancy makes Mutschler and colleagues' effort to identify a more reliable classification system particularly relevant to the successful management of the severely injured patient [1].

Base deficit has been frequently used to determine endpoints of resuscitation and has been predictive of both morbidity and mortality. As early as 1988, Davis and colleagues demonstrated a correlation between increasing 
base deficit and ongoing hemorrhage [9]. Since that time, multiple studies have shown a relationship between worsening base deficit and negative outcomes, including increased transfusion requirements, multiple organ failure, acute respiratory distress syndrome, and mortality [10]. In addition, the role of base-deficit measurement may take on new importance given the recent focus by the trauma community on the acute coagulopathy of trauma. A recent study by Sixta and colleagues demonstrated among operative trauma patients that base deficit was independently associated with the development of acute coagulopathy of trauma, an entity that contributes to ongoing hemorrhage [11]. Mutschler and colleagues' paper reflects several of these earlier findings but also appears to be the first study to define and validate a basedeficit-oriented classification system.

Despite its interesting conclusions, Mutschler and colleagues' paper does raise some questions regarding its applicability in the general trauma population [1]. The TraumaRegister $\mathrm{DGU}^{\circ}$ consisted largely of blunt trauma patients (92.3 to 96\%) and thus it is unclear how well the base-deficit classification system would function when applied to penetrating injuries. However, a recent article by Caputo and colleagues may provide some insight into this issue [12]. In Caputo and colleagues' study, there was no correlation between triage vital signs and lactate or base-deficit levels among penetrating trauma patients. However, serum lactate but not base deficit was correlated with the need for operative intervention. This study lends credence to the idea that vital signs are not reflective of shock in both penetrating and blunt injury but may call into question the applicability of base deficit in penetrating trauma. Owing to the small number of penetrating trauma patients examined in both studies, a true assessment cannot be determined without further study in a larger population.

Another concern regarding the use of base deficit is the need for invasive testing via the procurement of an arterial blood sample. Practitioners often deem it too difficult to obtain an arterial sample in the midst of triage activities and the results are often delayed by the need for laboratory analysis. However, the increased availability of point-of-care testing in emergency departments has significantly increased the speed with which blood gas results can be obtained. In addition, there is also some evidence that an arterial blood sample is not necessary and that a venous sample, which is routinely drawn during triage, can be used in its place. Arnold and colleagues compared base-deficit measurements between arterial and venous blood samples obtained from trauma patients in the emergency department [10]. The results obtained from the arterial and venous samples showed good correlation and the variations were within clinically acceptable ranges. Sample collection should therefore not significantly limit the use of base deficit in the classification of shock unless assessment is being performed in a resource-limited environment.

In conclusion, Mutschler and colleagues express a valid concern regarding the accuracy of the ATLS shock classification system and have offered a reasonable alternative [1]. Base-deficit measurement can be reasonably incorporated into the initial triage of trauma patients in all settings where point-of-care testing is readily available. Owing to the increasing availability of such tests and the significant benefits to be gained by earlier recognition of hypovolemic shock, the use of base deficit in shock classification should be further investigated and incorporated into the early triage and management of trauma patients.

Abbreviations

ATLS, Advanced Trauma Life Support.

\section{Competing interests}

The authors declare that they have no competing interests.

\section{Published: 13 March 2013}

\section{References}

1. Mutschler M, Neinaber U, Brockamp T, Wafaisade A, Fabian T, Paffrath T, Bouillon B, Maegel M; TraumaRegister DGU®: Renaissance of base deficit for the initial assessment of trauma patients: a base deficit-based classification for hypovolaemic shock developed on data from 16,305 patients derived from the TraumaRegister DGU ${ }^{\circledR}$. Crit Care 2013, 17:R42.

2. American College of Surgeons, Committee on Trauma: Advanced Trauma Life Support for Doctors - Student Course Manual. 8th edition. Chicago, IL: American College of Surgeons; 2008.

3. Guly HR, Bouamra O, Little R, Dark P, Coats T, Driscoll P, Lecky FE: Testing the validity of ATLS classification of hypovolaemic shock. Resuscitation 2010, 81:1142-1147.

4. Guly HR, Bouamra O, Spiers M, Dark P, Coats T, Lecky FE: Vital signs and estimated blood loss in patients with major trauma: testing the validity of the ATLS classification of hypovolaemic shock. Resuscitation 2011, 82:556-559.

5. Heckbert SR, Vedder NB, Hoffman W, Winn RK, Hudson LD, Jurkovich GJ, Copass MK, Harlan JM, Rice CL, Maier RV: Outcome after hemorrhagic shock in trauma patients. J Trauma 1998, 45:545-549.

6. Mutschler M, Nienaber U, Brockamp T, Wafaisade A, Wyen H, Peiniger S, Paffrath T, Bouillon B, Maegele M; TraumaRegister DGU ${ }^{\oplus}$ : A critical reappraisal of the ATLS classification of hypovolaemic shock: does it really reflect clinical reality? Resuscitation 2012, http://dx.doi.org/10.1016/j.resuscitation.2012.07.012

7. Ley EJ, Salim A, Kohanzadeh S, Mirocha J, Margulies DR: Relative bradycardia in hypotensive trauma patients: a reappraisal. J Trauma 2009, 67:1051-1054.

8. Mizushima Y, Ueno M, Watanabe H, Ishikawa K, Matsuoka T: Discrepancy between heart rate and markers of hypoperfusion is a predictor of mortality in trauma patients. J Trauma 2011, 71:789-792.

9. Davis JW, Shackford SR, Mackersie RC, Hoyt DB: Base deficit as a guide to volume resuscitation. J Trauma 1988, 28:1464-1467.

10. Arnold TD, Miller M, vanWessem KP, Evans JA, Balogh ZJ: Base deficit from the first peripheral venous sample: a surrogate for arterial base deficit in the trauma bay. J Trauma 2011, 71:793-797.

11. Sixta SL, Hatch QM, Matijevic N, Wade CE, Holcomb JB, Cotton BA: Mechanistic determinates of the acute coagulopathy of trauma (ACoT) in patients requiring emergency surgery. Int J Burn Trauma 2012, 2:158-166.

12. Caputo N, Fraser R, Paliga A, Kanter M, Hosford K, Madlinger R: Triage vital signs do not correlate with serum lactate or base deficit, and are less predictive of operative intervention in penetrating trauma patients: a prospective cohort study. Emerg Med J 2012, doi:10.1136/emermed-2012-201343

doi:10.1186/cc12513

Cite this article as: Privette AR, Dicker RA: Recognition of hypovolemic shock: using base deficit to think outside of the ATLS box. Critical Care 2013 , $17: 124$ 